\title{
RELATIONS BETWEEN WIENER, HYPER-WIENER AND SOME ZAGREB TYPE INDICES
}

\author{
Ali Ghalavand ${ }^{1 *}$, Mehdi Eliasi $^{2}$ and Ali Reza Ashrafi ${ }^{1}$ \\ ${ }^{1}$ University of Kashan, Faculty of Mathematical Sciences, Department of Pure Mathematics, \\ Kashan 87317-53153, I. R. Iran \\ ${ }^{2}$ University of Khansar, Department of Mathematics, Khansar, I. R. Iran \\ *Corresponding author; E-mail: alighalavand@grad.kashnu.ac.ir
}

(Received July 13th, 2018; Accepted August 20th, 2018)

\begin{abstract}
In this paper, some inequalities between the Wiener, hyper-Wiener, first Zagreb, second Zagreb, first reformulated Zagreb, second reformulated Zagreb and the general Zagreb indices of a simple graph are given. Our results improve some earlier bonds between these graph invariants.
\end{abstract}

Keywords: Topological index, Wiener index, Hyper-Wiener index, Zagreb indices, Reformulated Zagreb index.

\section{INTRODUCTION}

A graph is a pair $G=(V, E)$ in which $V$ is a non-empty set and $E \subseteq P_{2}(V)=$ $\{\{x, y\} \mid x \neq x \in V\}$. If each element of $V$ has at most four mates in $V$, then the graph $G$ is called a molecular graph. Topological indices are maps from molecular graphs into real numbers, such that this mapping is invariant under graph isomorphisms. These indices are widely used in chemistry for relationship between molecular structures and molecular properties of a given complex (DEVILLERS and BALABAN, 2000). The Wiener index (WIENER, 1947) and Zagreb group indices (GUTMAN and TRINAJSTIĆ, 1972; GUTMAN and FURTULA, 2003) are some of the most studied topological indices both by chemists and mathematicians. In a similar way as Zagreb group indices, the first and second reformulated Zagreb indices were defined (ILIĆ and ZHOU, 2012). In addition if $\alpha$ is an arbitrary real number except from 0 and 1 , then (LI and ZHENG, 2005) introduced the general Zagreb index of a graph. The most important generalization of Wiener index is the hyper-Wiener index (KLEIN et al., 1995). We will define these graph invariant later.

Let $G=(V, E)$ be a simple connected graph and $u, v \in V$. The distance between $u$ and $v, d_{G}(u, v)$, is defined as the minimum length of a shortest path connecting them. A graph invariant is said to be distance-based if it can be defined by distance function $d_{G}(-,-)$. The Wiener and hyper-Wiener indices of $G$ are defined as follows: 


$$
\begin{aligned}
& W(G)=\sum_{\{u, v\} \subset V(G)} d_{G}(u, v), \\
& W W(G)=\frac{1}{2}\left[W(G)+\sum_{\{u, v\} \subset V(G)} d_{G}(u, v)^{2}\right] .
\end{aligned}
$$

These are the most important distance-based topological indices of a graph.

Suppose $G$ is a simple graph and $v \in V(G)$. The notation $\operatorname{deg}(v)$ used for the degree of $v$ in $G$. A topological index is said to be degree-based if it can be defined as the function $\operatorname{deg}(-)$. The Zagreb and reformulated Zagreb group indices are most important degree-based topological indices of $G$. These topological indices, as well as, the general Zagreb index of $G$ are defined as follows:

$$
\begin{aligned}
& M_{1}(G)=\sum_{v \in V(G)} \operatorname{deg}_{G}(v)^{2} \& M_{2}(G)=\sum_{u v \in E(G)} \operatorname{deg}_{G}(u) \operatorname{deg}_{G}(v), \\
& E M_{1}(G)=\sum_{e \in E(G)} \operatorname{deg}_{G}(e)^{2} \& E M_{2}(G)=\sum_{e \sim f} \operatorname{deg}_{G}(e) \operatorname{deg}_{G}(f), \\
& M_{1}^{\alpha}(G)=\sum_{u \in V(G)} \operatorname{deg}_{G}(u)^{\alpha} .
\end{aligned}
$$

Here, $e \sim f$ means that the edges $e$ and $f$ have a common vertex, the notation $\operatorname{deg}_{G}(u)$ is used for the degree of vertex $v$ in $G$ and for an edge $e=u v, \operatorname{deg}_{G}(e)=$ $\operatorname{deg}_{G}(u)+\operatorname{deg}_{G}(v)-2$ denotes the degree of the edge $e$. It is easy to prove that $M_{1}(G)=$ $\sum_{u v \in E(G)}\left[\operatorname{deg}_{G}(u)+\operatorname{deg}_{G}(v)\right]$.

It is clear that $M_{1}^{2}(G)=M_{1}(G)$. The graph invariant $M_{1}^{3}(G)$ is called the forgotten topological index and denoted by $F(G) ; F(G)=\sum_{u \in V(G)} \operatorname{deg}_{G}(u)^{3}$ (FURTULA and GUTMAN, 2015). We encourage the interested readers to consult papers (GUTMAN et al., 1997; KLAVŽAR et al., 2000; NIKOLIĆ et al., 2003; XING et al., 2011) for more information on this topic.

It is natural to try to establish relations between the degree-based and distance-based topological indices. (ZHOU and GUTMAN, 2004) obtained some bounds on Wiener and hyper-Wiener indices, in terms of the first Zagreb index for molecular graphs with girth greater than four. BEHTOI et al. (2011) deduced inequalities for Wiener and hyper-Wiener indices, in terms of $M_{1}, M_{2}$ and the number of hexagons. DAS et al. (2015) continued the previous works by considering Szeged, PI, and Wiener polarity indices, as distance-based indices, and the first and second Zagreb indices.

The aim of this paper is to bring new inequalities, relating $W, W W, M_{1}, M_{2}, E M_{1}$, $E M_{2}, F$ and $M_{1}^{4}$. In addition our results correct some minor errors in previous works.

\section{PRELIMINARIES}

Let $G=(V(G), E(G))$ be a simple connected graph with $n$ vertices and $m$ edges, respectively. The diameter of $G$, denoted by $\operatorname{diam}(G)$, is defined as the largest distance between vertices of $G$. The length of a shortest cycle in $G$ is called the girth of $G$, denoted by $g(G)$. If $G$ does not contain a cycles, then we set $g(G)=\infty$. Let $k$ be a nonnegative integer. Then $d(G, k)$ denotes the number of pairs of vertices in $G$ with distance $k$. Note that $d(G, k)=0$, for every $k>\operatorname{diam}(G)$, and $d(G, 1)=m$. It is easy to check that

$$
\begin{aligned}
& \sum_{k \geq 1} d(G, k)=\left(\begin{array}{c}
n \\
2
\end{array}\right) \& W(G)=\sum_{k \geq 1} k d(G, k), \\
& W W(G)=\frac{1}{2} \sum_{k \geq 1} k(k+1) d(G, k) .
\end{aligned}
$$


ZHO and GutMAN (2004) showed that the equality $d(G, 2)=\frac{1}{2} M_{1}(G)-m$ holds for graphs that do not contain triangles and/or quadrangles; in fact $g(G)>4$. We may extend this result as:

Lemma 2.1 Let $G$ be a graph with $s$ squares, $m$ edges and $g(G) \geq 4$. Then $d(G, 2)=$ $\frac{1}{2} M_{1}(G)-m-2 s$.

Proof. Since $g(G) \geq 4$, for each square in $G$ there are exactly two pairs of vertices of distance two and hence two distinct paths of length two in $G$. It follows that

$d(G, 2)=\sum_{u \sim v \sim x} 1-2 s=\sum_{v \in V(G)} \operatorname{deg}_{G}(v)\left[\frac{\operatorname{deg}_{G}(v)-1}{2}\right] \quad-2 s=\frac{1}{2} M_{1}(G)-m-2 s$,

as desired.

BEHTOI et al. (2011) explained that if $g(G)>4$ and $G$ has $h$ hexagons, then $d(G, 3)=M_{2}(G)-M_{1}(G)+m-3 h$. Suppose $G$ is a cycle with 5 vertices, then $g(G)=5$ and $d(G, 3)=0$, but $M_{2}(G)-M_{1}(G)+m-3 h=10-10+5-3 \times 0=5$. In fact, $d(G, 3)=M_{2}(G)-M_{1}(G)+m-3 h$ holds when $g(G) \geq 6$, since we conclude from $g(G) \geq 6$ that each hexagon in $G$ has exactly three pairs of vertices of distance three and so two distinct paths of size three in $G$. Hence,

$$
\begin{aligned}
& d(G, 3)=\sum_{w \sim u \sim v \sim x} 1-3 h \\
& =\sum_{u \sim v}\left(\operatorname{deg}_{G}(u)-1\right)\left(\operatorname{deg}_{G}(v)-1\right)-3 h \\
& =\sum_{u \sim v}\left[\operatorname{deg}_{G}(u) \operatorname{deg}_{G}(v)-\left(\operatorname{deg}_{G}(u)+\operatorname{deg}_{G}(v)\right)+1\right]-3 h \\
& =M_{2}(G)-M_{1}(G)+m-3 h .
\end{aligned}
$$

This observation yields the following lemma;

Lemma 2.2 If $G$ is a graph with $h$ hexagons, $m$ edges and $g(G) \geq 6$, then $d(G, 3)=$ $M_{2}(G)-M_{1}(G)+m-3 h$.

We are now ready to extend this result to the case that $G$ has exactly $c$ octagons and $g(G) \geq 8$.

Lemma 2.3 Let $G$ be a graph with $c$ octagons, $n$ vertices and $m$ edges. If $A(G)=$ $\sum_{e \sim f, e=u v, f=v x}\left(\operatorname{deg}_{G}(v)-1\right)\left(\operatorname{deg}_{G}(e)+\operatorname{deg}_{G}(f)\right)$ and $g(G) \geq 8$, then $d(G, 4)=$ $E M_{2}(G)-A(G)+\frac{1}{2} M_{1}^{4}(G)-\frac{3}{2} F(G)+\frac{3}{2} M_{1}(G)-m-4 c$.

Proof. Since $g(G) \geq 8$, for each octagon in $G$ there are exactly four pairs of vertices of distance four and hence two distinct paths of length four in $G$. Therefore,

$$
\begin{aligned}
& d(G, 4)=\sum_{w \sim u \sim v \sim x \sim z} 1-4 c \\
& =\sum_{u \sim v \sim x}\left(\operatorname{deg}_{G}(u)-1\right)\left(\operatorname{deg}_{G}(x)-1\right)-4 c \\
& =\sum_{e \sim f, e=u v, f=v x}\left(\operatorname{deg}_{G}(e)-\left(\operatorname{deg}_{G}(v)-1\right)\right)\left(\operatorname{deg}_{G}(f)-\left(\operatorname{deg}_{G}(v)-1\right)\right)-4 c \\
& =\sum_{e \sim f, e=u v, f=v x}\left[\operatorname{deg}_{G}(e) \operatorname{deg}_{G}(f)-\operatorname{deg}_{G}(e)\left(\operatorname{deg}_{G}(v)-1\right)\right. \\
& \left.-\left(\operatorname{deg}_{G}(v)-1\right) \operatorname{deg}_{G}(f)+\left(\operatorname{deg}_{G}(v)-1\right)^{2}\right]-4 c \\
& =\sum_{e \sim f, e=u v, f=v x} \operatorname{deg}_{G}(e) \operatorname{deg}_{G}(f)+\sum_{e \sim f, e=u v, f=v x}\left(\operatorname{deg}_{G}(v)-1\right)^{2}-4 c \\
& -\sum_{e \sim f, e=u v, f=v x}\left(\operatorname{deg}_{G}(v)-1\right)\left(\operatorname{deg}_{G}(e)+\operatorname{deg}_{G}(f)\right) .
\end{aligned}
$$


So, by definition,

$$
\begin{aligned}
& d(G, 4)=E M_{2}(G)-\sum_{e \sim f, e=u v, f=v x}\left(\operatorname{deg}_{G}(v)-1\right)\left(\operatorname{deg}_{G}(e)+\operatorname{deg}_{G}(f)\right) \\
& +\sum_{v \in V(G)}\left(\operatorname{deg}_{G}(v)\right)\left(\operatorname{deg}_{G}(v)-1\right)^{2}-4 c \\
& =E M_{2}(G)-\sum_{e \sim f, e=u v, f=v x}\left(\operatorname{deg}_{G}(v)-1\right)\left(\operatorname{deg}_{G}(e)+\operatorname{deg}_{G}(f)\right) \\
& +\frac{1}{2} \sum_{v \in V(G)}\left[\operatorname{deg}_{G}^{4}(v)-3 \operatorname{deg}_{G}^{3}(v)+3 \operatorname{deg}_{G}^{2}(v)-\operatorname{deg}_{G}(v)\right]-4 c \\
& =E M_{2}(G)-\sum_{e \sim f, e=u v, f=v x}\left(\operatorname{deg}_{G}(v)-1\right)\left(\operatorname{deg}_{G}(e)+\operatorname{deg}_{G}(f)\right) \\
& +\frac{1}{2} M_{1}^{4}(G)-\frac{3}{2} F(G)+\frac{3}{2} M_{1}(G)-m-4 c \\
& =E M_{2}(G)-A(G)+\frac{1}{2} M_{1}^{4}(G)-\frac{3}{2} F(G)+\frac{3}{2} M_{1}(G)-m-4 c .
\end{aligned}
$$

This completes the proof.

From the previous lemma, we conclude that:

Corollary 2.4 Let $G$ be a graph with $c$ octagons, $n$ vertices, $m$ edges and $g(G) \geq 8$. Then $\sum_{k \geq 5} d(G, k)=\frac{n(n-1)}{2}-\left[M_{1}(G)+M_{2}(G)+E M_{2}(G)-A(G)+\frac{1}{2} M_{1}^{4}(G)-\frac{3}{2} F(G)-4 c\right]$.

\section{MAIN RESULTS}

Suppose $G$ is a connected simple graph with $n$ vertices and $m$ edges. Then the Wiener, hyper-Wiener, Zagreb indices and general Zagreb indices satisfy the following relations:

Theorem 3.1 Suppose that $G$ is a graph with $c$ octagons, $n$ vertices, $m$ edges and $g(G) \geq 8$. If $\delta^{\prime}(G)=\delta^{\prime}=\min \left\{\operatorname{deg}_{G}(v) \mid v \in V(G) \operatorname{anddeg}_{G}(v) \neq 1\right\}$, then

1. $W(G) \geq \frac{5 n(n-1)}{2}+\frac{3}{2} F(G)+\left(\delta^{\prime}-1\right) E M_{1}(G)+4 c-\left(2 m+E M_{2}(G)+\right.$ $\left.M_{1}(G)+2 M_{2}(G)+\frac{1}{2} M_{1}^{4}(G)\right)$,

2. $W W(G) \geq \frac{1}{2} W(G)+\frac{1}{2}\left[\frac{25 n(n-1)}{2}+\frac{27}{2} F(G)+9\left(\delta^{\prime}-1\right) E M_{1}(G)+36 c-\right.$ $\left.\left(10 m+9 E M_{2}(G)+8 M_{1}(G)+16 M_{2}(G)+\frac{9}{2} M_{1}^{4}(G)\right)\right]$,

3. $W W(G) \geq \frac{1}{2}\left[15 n(n-1)+15 F(G)+10\left(\delta^{\prime}-1\right) E M_{1}(G)+40 c-(12 m+\right.$ $\left.\left.10 E M_{2}(G)+9 M_{1}(G)+18 M_{2}(G)+5 M_{1}^{4}(G)\right)\right]$,

4. $W W(G) \geq 3 W(G)+3 F(G)+2\left(\delta^{\prime}-1\right) E M_{1}(G)+8 c-\left(2 E M_{2}(G)+\right.$ $\left.\frac{3}{2} M_{1}(G)+3 M_{2}(G)+M_{1}^{4}(G)\right)$.

The equality in all four cases will hold if and only if the distance between any two vertices in $G$ is not greater than 5 and $\left\{\operatorname{deg}_{G}(v) \mid v \in V(G)\right.$ and $\left.\operatorname{deg}_{G}(v) \neq 1\right\}=\left\{\delta^{\prime}\right\}$.

Proof. From what has already been proved in section 2, we have:

$\sum_{k \geq 5} k d(G, k)$

(1) $W(G)=\sum_{k \geq 1} k d(G, k)=d(G, 1)+2 d(G, 2)+3 d(G, 3)+4 d(G, 4)+$

$$
\begin{aligned}
& \geq m+M_{1}(G)-2 m+3 M_{2}(G)-3 M_{1}(G)+3 m+4 E M_{2}(G)-4 A(G) \\
& +2 M_{1}^{4}(G)-6 F(G)+6 M_{1}(G)-4 m-16 c+5 \sum_{k \geq 5} d(G, k) \\
& =m+M_{1}(G)-2 m+3 M_{2}(G)-3 M_{1}(G)+3 m+4 E M_{2}(G)-4 A(G) \\
& +2 M_{1}^{4}(G)-6 F(G)+6 M_{1}(G)-4 m-16 c+\frac{5 n(n-1)}{2}-5 M_{1}(G) \\
& -5 M_{2}(G)-5 E M_{2}(G)+5 A(G)-\frac{5}{2} M_{1}^{4}(G)+\frac{15}{2} F(G)+20 c \\
& \geq \frac{5 n(n-1)}{2}+\frac{3}{2} F(G)+\left(\delta^{\prime}-1\right) E M_{1}(G)+4 c
\end{aligned}
$$




$$
-\left(2 m+E M_{2}(G)+M_{1}(G)+2 M_{2}(G)+\frac{1}{2} M_{1}^{4}(G)\right) .
$$

The last inequality follows from $A(G) \geq\left(\delta^{\prime}-1\right) E M_{1}(G)$.

$$
\begin{aligned}
& (2) W W(G)=\frac{1}{2} \sum_{k \geq 1} k(k+1) d(G, k)=\frac{1}{2} W(G)+\frac{1}{2} \sum_{k \geq 1} k^{2} d(G, k) \\
= & \\
\frac{1}{2} W(G)+\frac{1}{2}[ & \left.d(G, 1)+4 d(G, 2)+9 d(G, 3)+16 d(G, 4)+\sum_{k \geq 5} k^{2} d(G, k)\right] \\
\geq & \frac{1}{2} W(G)+\left[\frac{m}{2}+M_{1}(G)-2 m+\frac{9}{2} M_{2}(G)-\frac{9}{2} M_{1}(G)+\frac{9}{2} m+8 E M_{2}(G)\right. \\
& -8 A(G)+4 M_{1}^{4}(G)-12 F(G)+13 M_{1}(G)-8 m-32 c+\frac{25}{2} \sum_{k \geq 5} d(G, k) \\
& =\frac{1}{2} W(G)-5 m+\frac{9}{2} M_{2}(G)-\frac{9}{2} M_{1}(G)+8 E M_{2}(G)-8 A(G) \\
& +4 M_{1}^{4}(G)-12 F(G)+12 M_{1}(G)+18 c+\frac{25 n(n-1)}{4}+\frac{75}{4} F(G) \\
& -\frac{25}{2} M_{1}(G)-\frac{25}{2} M_{2}(G)-\frac{25}{2} E M_{2}(G)+\frac{25}{2} A(G)-\frac{25}{4} M_{1}^{4}(G) \\
& \geq \frac{1}{2} W(G)+\frac{25 n(n-1)}{4}+\frac{27}{4} F(G)+\frac{9}{2}\left(\delta^{\prime}-1\right) E M_{1}(G)+18 c \\
& -\left(5 m+\frac{9}{2} E M_{2}(G)+4 M_{1}(G)+8 M_{2}(G)+\frac{9}{4} M_{1}^{4}(G)\right)
\end{aligned}
$$

in which the last inequality follows from $A(G) \geq\left(\delta^{\prime}-1\right) E M_{1}(G)$. The inequality (3) is a direct consequence of (1) and (2).

$$
\begin{aligned}
& (4) W W(G)=\frac{1}{2} \sum_{k \geq 1} k(k+1) d(G, k)=\frac{1}{2} W(G)+\frac{1}{2} \sum_{k \geq 1} k^{2} d(G, k) \\
& = \\
\frac{1}{2} W(G)+\frac{1}{2}[ & \left.(G, 1)+4 d(G, 2)+9 d(G, 3)+16 d(G, 4)+\sum_{k \geq 5} k^{2} d(G, k)\right] \\
& \geq \frac{1}{2} W(G)+M_{1}(G)-5 m+\frac{9}{2} M_{2}(G)+\frac{15}{2} M_{1}(G)+8 E M_{2}(G)-8 A(G) \\
& +4 M_{1}^{4}(G)-12 F(G)-32 c+\frac{5}{2} \sum_{k \geq 5} k d(G, k) \\
& =\frac{1}{2} W(G)-5 m+\frac{17}{2} M_{1}(G)+\frac{9}{2} M_{2}(G)+8 E M_{2}(G) \\
& -8 A(G)+4 M_{1}^{4}(G)-12 F(G)-32 c \\
& +\frac{5}{2}[W(G)-(d(G, 1)+2 d(G, 2)+3 d(G, 3)+4 d(G, 4))] \\
& =3 W(G)-5 m+\frac{17}{2} M_{1}(G)+\frac{9}{2} M_{2}(G)+8 E M_{2}(G) \\
& -8 A(G)+4 M_{1}^{4}(G)-12 F(G)-32 c \\
& +5 m-10 M_{1}(G)-\frac{15}{2} M_{2}(G)-10 E M_{2}(G) \\
& +10 A(G)-5 M_{1}^{4}(G)+15 F(G)+40 c \\
& =3 W(G)+3 F(G)+2 A(G)+8 c \\
& -\left(2 E M_{2}(G)+\frac{3}{2} M_{1}(G)+3 M_{2}(G)+M_{1}^{4}(G)\right) \\
\geq & 3 W(G)+3 F(G)+2\left(\delta^{\prime}-1\right) E M_{1}(G)+8 c \\
& -\left(2 E M_{2}(G)+\frac{3}{2} M_{1}(G)+3 M_{2}(G)+M_{1}^{4}(G)\right)
\end{aligned}
$$

in which the last inequality is a consequence of $A(G) \geq\left(\delta^{\prime}-1\right) E M_{1}(G)$. Equality in $(1-4)$ hold if and only if $\sum_{k \geq 5} k d(G, k)=5 \sum_{k \geq 5} d(G, k)\left(\right.$ or $\left.\sum_{k \geq 5} k^{2} d(G, k)=25 \sum_{k \geq 5} d(G, k)\right)$ and $A(G)=\left(\delta^{\prime}-1\right) E M_{1}(G)$. Therefore, these equalities can be occurred if and only if the distance between any two vertices of the graph $G$ is not greater than 5 and $\left\{\operatorname{deg}_{G}(v) \mid v \in\right.$ $V(G)$ and $\left.\operatorname{deg}_{G}(v) \neq 1\right\}=\left\{\delta^{\prime}\right\}$.

\section{Acknowledgments}

The research of the first and third authors are partially supported by the University of Kashan under grant no 364988/489. 


\section{References:}

[1] Behtoei, A., Jannesari, M., TAeri, B. (2011): Some new relations between Wiener, hyper-Wiener and Zagreb indices. MATCH Communicationsin Mathematical and in Computer Chemistry 65(1): 27-32.

[2] Devillers, J., Balaban, A.T. (2000): Topological Indices and Related Descriptors in QSAR and QSPAR. United Kingdom, Taylor \& Francis Ltd.

[3] Das, K.Ch., Gutman, I., NADJAFI-ARAni, M.J. (2015): Relations between distance-based and degree-based topological indices. Applied Mathematics and Computation 270: 142-147. doi: 10.1016/j.amc.2015.08.061

[4] Das, K.Ch., JeOn, H., Trinajstić, N. (2014): Comparison between the Wiener index and the Zagreb indices and the eccentric connectivity index for trees. Discrete Applied Mathematics 171: 35-41. doi: 10.1016/j.dam.2014.02.022

[5] Dobrynin, A.A., Entringer, R., Gutman, I. (2001): Wiener index of trees: Theory and applications. Acta Applicandae Mathematica 68 (3): 211-249. doi: 10.1023/A:10107675 17079

[6] Furtula, B., Gutman, I. (2015): A forgotten topological index. Journal of Mathematical Chemistry 53 (4): 1184-1190. doi: 10.1007/s10910-015-0480-z

[7] Gutman, I., Trinajstić, N. (1972): Graph theory and molecular orbitals. Total $\pi$-electron energy of alternant hydrocarbons. Chemical Physics Lettetters 17 (4): 535-538. doi: 10.1016/0009-2614(72)85099-1

[8] Gutman, I., Furtula, B. (2003): Hyper-Wiener index vs. Wiener index. Two highly correlated structure-descriptors. Monatshefte für Chemie 134(7): 975-981. doi: 10.1007/s 00706-003-0003-7

[9] Gutman, I., Linert, W., Lukovits, I., Dobrynin, A.A. (1997): Trees with extremal hyper-Wiener index: Mathematical basis and chemical applications. Journal of Chemical Information and Computer Sciences 37(2): 349-354. doi: 10.1021/ci960139m

[10] Ilić, A., Zhou, B. (2012): On reformulated Zagreb indices. Discrete Applied Mathematics 160(3): 204-209. doi: 10.1016/j.dam.2011.09.021

[11] KlavŽAR, S., Zigert, P., GutMan, I. (2000): An algorithm for the calculation of the hyper-Wiener index of benzenoid hydrocarbons. Computers \& Chemistry 24(2): 229-233. doi: 10.1016/S0097-8485(99)00062-5

[12] Klein, D.J., Lukovits, I., GutMan, I. (1995): On the definition of the hyper-Wiener index for cycle-containing structures. Journal of Chemical Information and Computer Sciences 35(1): 50-52. doi: 10.1021/ci00023a007

[13] LI, X., ZHENG, J. (2005): A unified approach to the extremal trees for different indices. MATCH Communications in Mathematical and in Computer Chemistry 54 (1): 195-208.

[14] Nikolić, S., KovaČević, G., MiličEvić, A., Trinajstić, N. (2003): The Zagreb indices 30 years after. Croatica Chemica Acta 76(2): 113-124.

[15] WIENER, H. (1947): Structural determination of paraffin boiling points. Journal of the Americal Chemical Society 69(1): 17-20. doi: 10.1021/ja01193a005

[16] XING, R., ZHOU, B., QI, X. (2011): Hyper-Wiener index of unicyclic graphs. MATCH Communications in Mathematical and in Computer Chemistry 66(1): 315-328.

[17] Zhou, B., Gutman, I. (2004): Relations between Wiener, hyper-Wiener and Zagreb indices. Chemical Physics Letters 394(1-3): 93-95. doi: 10.1016/j.cplett.2004.06.117 